\title{
Moldavie et langue française, une relation séculaire et privilégiée
}

Olga Turcan

\section{(2) OpenEdition \\ 1 Journals}

Édition électronique

URL : http://journals.openedition.org/esp/774

DOI : $10.4000 /$ esp. 774

ISSN : 2532-0319

Éditeur

Centre d'Information sur l'Éducation Bilingue et Plurilingue

\section{Édition imprimée}

Date de publication : 1 juin 2016

Pagination : 15-26

ISSN : 1127-266X

\section{Référence électronique}

Olga Turcan, « Moldavie et langue française, une relation séculaire et privilégiée », Éducation et sociétés plurilingues [En ligne], 40 | 2016, mis en ligne le 28 octobre 2016, consulté le 03 mai 2019. URL : http:// journals.openedition.org/esp/774 ; DOI : 10.4000/esp.774 


\section{MOLDAVIE ET LANGUE FRANÇAISE, UNE RELATION SÉCULAIRE ET PRIVILÉGIÉE}

\section{Olga Turcan}

Questo lavoro analizza il posto del francese in Moldavia prima e dopo l'indipendenza ottenuta nel 1991, nei contesti dell'eredità culturale, della tradizione dell'insegnamento e della globalizzazione. Prende in esame l'esistenza di una politica linguistica nei confronti del francese e la posizione dei cittadini nei confronti di questa politica. Questo studio si basa su un corpus costituito da documenti d'archivio (1975-2010), da un'inchiesta socio-linguistica (2009), da regolamentazioni recenti (2011-2013), da colloqui con i protagonisti del sistema educativo e della francofonia in Moldavia, da articoli di media e da pubblicazioni statistiche. Lo studio incrociato delle analisi di discorsi socio-educativi e dei cambiamenti di prospettiva socio-economica permette di ottenere elementi in grado di chiarire les problematiche abbordate.

Parole chiave: Lingua francese, politica linguistica, francofonia, Moldavia, discorso, rappresentazioni

Retracing the history of today's Republic of Moldavia, the article studies the place occupied by French in the country before and after its Independence in 1991, in between inherited and ingrained cultural habits, scholarly tradition and globalization. It examines the existence of a language policy concerning French and citizens' attitudes towards that policy. It is based on a body of archives (1975-2010), a sociolinguistic survey (2009), recent legal texts (2011-2013), interviews with actors of the education system and Francophony in Moldavia, and press articles as well as statistics. Correlations between socio-educational discourses and changes in the socio-economic perspective permit us to shed some light on the problems that emerge.

Key-words: French, language policy, Francophony, Moldavia, discourse, representations

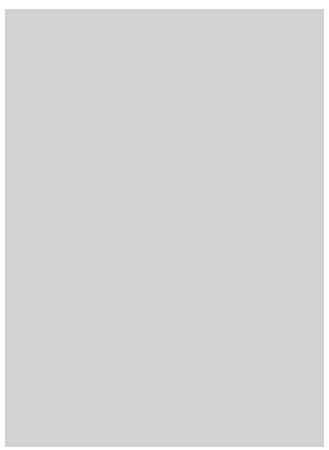

Yet article est un aperçu de la situation du français en Moldavie avant et après 1991, année de son indépendance (Turcan 2014). Nous exposerons les grandes lignes des principaux changements politiques traversés par la Moldavie qui ont influencé sa situation linguistique, y compris celle de la langue française «importée» sur son territoire. Claude Truchot fait observer que «la situation du français à l'époque contemporaine dans ces pays [la Moldavie, la Roumanie, ...] est encore peu étudiée» (Truchot 2000: 73).

Le nom de «Moldavie» renvoie ici au territoire de l'actuelle République de Moldavie (ou Moldova), pays frontalier de la 
Moldavie et langue française, une relation séculaire et privilégiée

O. TURCAN

\section{MÉTHODE ET} DONNÉES
Roumanie et de l'Ukraine. À ne pas confondre avec la Moldavie (roumaine) qui fait actuellement partie de la Roumanie, État membre de l'Union Européenne depuis 2007.

Dans le passé, ces deux «Moldavie» formaient la Principauté de Moldavie, apparue sur la carte politique de l'Europe en 1359. Leur séparation a eu lieu en 1812, à l'issu de la guerre russo-turque, lorsque la partie orientale de la principauté de Moldavie est rattachée à l'Empire russe et devient une de ses provinces, appelée également «Bessarabie». Cette dénomination est encore beaucoup utilisée et permet de faire formellement la distinction entre la Moldavie roumaine (région de la Roumanie) et la République de Moldavie.

Après un siècle de domination russe, lorsque le tsar abdique en 1917 et le mouvement des bolchéviques prend de l'ampleur, dans la goubernie de Bessarabie se forme le Conseil du pays (en roumain, Sfatul Țării). En janvier 1918, cette assemblée vote à l'unanimité l'indépendance de la République démocratique de Moldavie et deux mois plus tard, son union avec la Roumanie. Le territoire de 9 districts de la Bessarabie fait partie de la Grande Roumanie (România Mare) jusqu'en 1940. Dans cette période, la langue roumaine retrouve son statut de langue de l'administration et de l'enseignement en Bessarabie, statut qu'elle avait perdu pendant la domination russe.

En 1940, suite à l'entente entre Hitler et Staline sur les sphères d'influence transposée dans le pacte germano-soviétique «de nonagression» en août 1939 et au traité de Vienne de 1940, la Bessarabie est cédée à l'URSS et devient pendant environ un demi-siècle la République socialiste soviétique moldave, RSSM.

Le 27 août 1991, après le démantèlement de l'URSS, le Parlement moldave adopte la Déclaration de l'Indépendance de la République de Moldavie (Moldova) qui, proclamant l'indépendance de l'État démocratique - désormais République de Moldavie - sollicite la reconnaissance internationale et son adhésion à l'Organisation des Nations Unies (mars 1992).

Les régions moldaves de «Transnistrie» (le territoire entre le fleuve Dniestr et la frontière internationalement reconnue entre la République de Moldavie et l'Ukraine) et de «Gagaouzie», situées près de la frontière sud avec l'Ukraine, autoproclament leur souveraineté en septembre 1990. Le Parlement de la République de Moldavie a officiellement reconnu l'autonomie de la Gagaouzie en décembre 1994, mais n'a jamais reconnu, comme d'ailleurs la communauté internationale, la «République moldave du Dniestr» 
Moldavie et langue française, une relation séculaire et privilégiée

O. TURCAN

\section{LA SITUATION LINGUISTIQUE GÉNÉRALE}

\section{LE FRANÇAIS AVANT} ET APRÈS 1991
[Transnistrie] avec sa capitale à Tiraspol, ni eu le contrôle de ce territoire. En Gagaouzie (officiellement «unité territoriale autonome de Gagaouzie», UTAG), à côté des Moldaves, habitent des Bulgares et des Gagaouzes (peuple turcophone chrétien) qui y sont installés après le rattachement à l'Empire russe, en 1812.

En 2015, la Moldavie compte 3,5 millions d'habitants, selon les statistiques officielles, dont un quart environ habite la capitale, la ville de Chisinau, et ses alentours.

Le roumain constitue la seule «langue d'État» de la Moldavie (Constitution de 1994, la Déclaration d'Indépendance de la Moldavie). Dans la majorité d'établissements scolaires en Moldavie, l'enseignement est dispensé en roumain. Les ethnies russe, ukrainienne, bulgare, juive, etc., habitant en Moldavie se voient accorder le droit d'utiliser leur langue, dans l'enseignement principalement. L'UTAG utilise sur son territoire le gagaouze, le russe et le roumain dans la sphère publique; la région de Transnistrie emploie le russe, après 1992, malgré la prédominance des Moldaves sur ce territoire. En dehors de la région transnistrienne, l'emploi du russe en Moldavie domine celui des autres langues des minorités et dans plusieurs cas les remplace, en tant que langue de «communication interethnique sur le territoire de la république», à côté du roumain. Cette situation du russe est héritée de la période soviétique en particulier. En dehors des pratiques sociales, la sphère administrative en commençant par les structures étatiques de haut niveau (parlement, gouvernement) élabore souvent les documents officiels en deux versions, roumain et russe.

Au sujet des langues dites étrangères enseignées en Moldavie, en 2007, 60\% des élèves apprenaient le français à l'école comme langue étrangère principale. L'anglais occupait la deuxième place loin devant les autres langues principalement enseignées en Moldavie (l'allemand, l'espagnol et l'italien, par ordre décroissant selon le nombre d'apprenants dans le primaire et le secondaire). Le questionnement sur la place importante occupée par le français en Moldavie, à une époque où les pays voisins et l'Europe en général étaient déjà depuis longtemps tournés vers l'anglais, a déterminé le choix de notre sujet de thèse «Le français en Moldavie», dont cet article donne un petit aperçu.

Le discours justifiant la place dominante du français dans l'enseignement des langues étrangères en Moldavie se réfère à l'héritage historique. Appuyé sur cet héritage, un autre argument courant 
Moldavie et langue française, une relation séculaire et privilégiée

O. TURCAN consiste à s'appuyer sur le fait qu'il est de tradition de l'enseigner. Nous avons interrogé cet héritage et nos recherches nous ont fait remonter au $18^{\mathrm{e}}$ siècle, lorsque la langue française a connu une diffusion dans toute l'Europe. Le questionnement sur les origines de la tradition d'enseignement du français nous a incitée à examiner les mesures de la part des autorités éducatives moldaves qui auraient pu la favoriser, tout cela dans le cadre plus large d'une éventuelle politique linguistique à l'égard du français avant et après 1991 (Klinkenberg 2000).

Dans la période avant 1991 le français garde une position dominante en Moldavie grâce à l'«héritage historique» et à la «tradition» d'enseignement. L'héritage historique se rapporte à la période d'avant 1940 (l'année du rattachement à l'URSS) et la tradition se réfère à la période qui lui succède jusqu'à aujourd'hui.

$\mathrm{Au} 18^{\mathrm{e}}$ siècle, tant en Moldavie que dans toute l'Europe, c'est la classe dominante qui adopte le français. Les princes phanariotes et les familles de nobles roumains se focalisent autour du français considéré non seulement comme «symbole de culture» et outil de communication, mais aussi et surtout un instrument de pouvoir, un signe de distinction et une marque de prestige. Son statut symbolique de langue universelle s'étend et se réduit en même temps à une élite au pouvoir, privilégiée et riche. De nombreux acteurs ont contribué à la diffusion du français sur ce territoire: les Phanariotes (Roumains d'ascendance hellénique), les Français - de passage ou émigrés - les enseignants de français de différentes origines, les Roumains eux-mêmes, les Russes francisés. Nombreux sont les secteurs qui l'adoptent et le font circuler: éducation, théâtre, presse, commerce, armée, diplomatie, littérature...

Une continuité peut être observée dans la diffusion du français au $19^{\mathrm{e}}$ siècle, où il garde encore son statut de langue élitaire, tout en devenant l'apanage de la couche sociale émergeante: l'intelligentsia. Les intellectuels, issus des familles nobles, pour partie, la parlent ou l'emploient dans leurs produits ou créations littéraires, scientifiques ou culturelles.

L'enseignement du français est dispensé à Chisinau et dans d'autres villes au niveau secondaire laïc ou religieux (écoles, collèges, gymnases, pensions, séminaires). Le français figure parmi les matières obligatoires et facultatives (l'allemand et le russe étaient également étudiés, le plus grand nombre d'heures étant accordé au russe). Les émigrés de différentes origines installés en Bessarabie, dont les «Français au service de la Russie» (Salon 
Moldavie et langue française, une relation séculaire et privilégiée

O. TURCAN
1981: 96-97), l'y enseignaient. Plus tard, cet enseignement sera fait par les autochtones. Ceux qui souhaitent continuer à l'apprendre ou le parfaire partent à Odessa, à Iassi (deux villes avec une présence française croissante au $19^{\mathrm{e}}$ siècle), à Bucarest ou à Paris même, car les voyages et les études en France se pratiquent de plus en plus fréquemment.

$\mathrm{Au} 20^{\mathrm{e}}$ siècle, lorsque la Moldavie fait partie de la Grande Roumanie (1918-1940), les données recueillies montrent que le développement de l'enseignement roumain, privé et public, a été propice à la diffusion du français. Dans les écoles moldaves le français continue à être enseigné, à côté du latin et de l'allemand. L'étude de la période 1940-1991 permet de constater que le français occupe une place importante parmi les autres langues (allemand, anglais, espagnol, italien). Les documents d'archives du Ministère de l'Éducation sur la création des premières facultés de langues étrangères dans les années 1940 mettent en évidence l'Institut pédagogique A. Russo de Balti: il ressort qu'il était la principale institution moldave à enjeux importants dans la formation de professeurs de français en Moldavie et en URSS et, par conséquent, dans la diffusion ultérieure du français dans les écoles moldaves. Cette institution d'enseignement supérieur aurait été le plus grand centre de l'URSS où l'on formait les professeurs de français, avec plus de 250 étudiants. Les premières promotions de diplômés ont fait émerger par la suite des professeurs de renom, auteurs de manuels, pionniers dans l'enseignement de plusieurs domaines disciplinaires de la linguistique française dans le milieu universitaire de Moldavie (Gutu 2006: 37-40). Les documents d'archives contiennent les indications d'une volonté politique d'accorder et de maintenir une place importante au français dans l'enseignement supérieur. Sachant que le Ministère de l'Éducation avait obligation de garantir ultérieurement un emploi aux jeunes diplômés, les postes d'enseignants de français étaient en conséquence plus nombreux et le nombre d'élèves plus important également, contribuant à instituer une tradition d'enseignement du français.

Quelles étaient les raisons de la continuité de la diffusion prédominante du français en Moldavie à l'époque où Moscou favorisait plutôt l'enseignement de l'allemand et de l'anglais? Une réponse possible serait l'héritage historique et une volonté politique de maintenir le français dans le seul pays soviétique de langue latine, et dans le cadre d'une politique conjoncturelle, où Moscou s'engage devant la France à augmenter le nombre d'élèves du français. Les données indiquent que $72 \%$ des élèves moldaves apprennent le 
Moldavie et langue française, une relation séculaire

et privilégiée

O. TURCAN

\section{LE RECUL QUANTITATIF DU FRANÇAIS APRÈS 1991}

français en 1981, et jusqu'en 1991. Avant 1991, une seule langue étrangère était généralement enseignée à côté du roumain et du russe.

1991 (année de l'indépendance) est un tournant dans l'enseignement des langues étrangères, dû à la politique internationale d'ouverture. La L2 est introduite dans l'enseignement secondaire. La langue française tombe sous le coup des mesures générales à l'égard des langues, sans être spécialement visée par les objectifs du Ministère. Dans leurs discours en 1993, les décideurs mentionnent l'intention de renforcer l'anglais. L'absence de soutien à l'égard du français et le soutien actif à l'anglais détermine dans la période de 1991 à 2011 le recul quantitatif progressif du français et un progrès stable de l'anglais. Dans cet intervalle, où le français régresse quantitativement mais reste tout de même en tête du classement, deux projets majeurs en sa faveur ont vu le jour: les filières universitaires francophones dans le supérieur (1997) et les classes bilingues dans le primaire et secondaire (1998). L'Agence universitaire de la Francophonie (AUF), en partenariat avec le Ministère des Affaires étrangères français et des acteurs institutionnels moldaves intervenant à différentes étapes (financement, formation, logistique et ressources humaines, évaluation,...), a mis en place les classes bilingues français-roumain à profil scientifique (primaire-collège-lycée). Ont également été créées les filières universitaires francophones professionnalisantes (industrie alimentaire, médecine, droit, économie, gestion, informatique et relations économiques internationales). Les enjeux pour le français sont au moins doubles: augmentation (même si ce n'est pas de manière substantielle) du nombre des francophones moldaves, mais aussi et surtout renforcement de la qualité de l'enseignement-apprentissage du français et de son usage. Ces parcours ouvrent également une porte vers les universités françaises, principalement pour la mobilité des professeurs, élèves ou étudiants et pour la poursuite d'études de master ou doctorat.

À côté des classes bilingues roumain-français, il y a également des écoles qui proposent l'étude approfondie du français. Il s'agit d'un nombre d'heures plus élevé accordé au français que dans les écoles «classiques», sans la possibilité d'étudier d'autres disciplines [scientifiques] en français. L'enseignement approfondi du français y est aussi plus répandu, car il est proposé tant dans les écoles de langue roumaine que dans les écoles de langue russe. Le lycée roumain-français Gh. Asachi de Chisinau propose à la fois les classes bilingues et l'enseignement renforcé du français dès le pri- 
Moldavie et langue française, une relation séculaire et privilégiée

O. TURCAN
EN 2011, LE FRANÇAIS PASSE AU 2E RANG maire. Il existe d'ailleurs une variation géographique de la place du français. Dans les grandes villes (Chisinau, Balti) c'est l'anglais qui domine. Dans le milieu rural habité par $66 \%$ de la population moldave, c'est le français qui garde souvent une position dominante.

Dans l'UTAG et la Transnistrie, c'est l'anglais qui se place en tête du classement. Cela est dû au lien entre la langue d'enseignement, le russe, et la langue étrangère, l'anglais. En effet, on observe une corrélation entre l'enseignement en roumain et les langues romanes comme le français principalement, l'espagnol et l'italien et une corrélation entre l'enseignement en russe et celui des langues germaniques comme l'anglais ou l'allemand. En 2011-2012, environ $80 \%$ du total d'élèves font leurs études en roumain et $20 \%$ en russe. Il s'agirait d'une loi non écrite, tacite, dans l'enseignement moldave: le critère d'une certaine proximité linguistique déterminerait l'enseignement d'une langue étrangère à l'école. Suivant cette logique, le français était généralement enseigné dans les écoles où la langue d'enseignement était le roumain et l'anglais dans les écoles en russe. Garder ce mode opératoire (corrélation roumain-français, russe-anglais) relèverait plus d'habitudes difficiles à modifier que d'un choix volontaire.

Il est à noter que dans le «Curriculum de Français pour les classes bilingues» (2008), les auteurs mentionnent un nouveau statut du français dans l'enseignement moldave, celui de «français langue seconde», FLS (qui concerne environ $2 \%$ des élèves qui apprennent le français en Moldavie) vs le français langue étrangère (FLE). Cependant, peut-on parler de FLS dans le contexte des classes bilingues moldaves où le français est appris de manière approfondie (de 3 à 5 heures de français par semaine) et où quatre autres matières sont apprises en français, mais en l'absence d'une pratique dans un «environnement quotidien spontané» (Blanchet 2012: 164)? La question est posée.

En 2011, vingt après l'indépendance, se produit l'inversion des statistiques en faveur de l'anglais. C'est la première fois que l'anglais passe en tête de classement, même si, en termes quantitatifs, le nombre de ses apprenants ne dépasse pas beaucoup celui du français, car il s'agit d'un peu plus de 7000 élèves.

Pour les années suivantes, si l'on regarde du côté des statistiques pour la période 2011-2013, et notamment le pourcentage d'élèves étudiant le français et l'anglais dans le primaire et le secondaire, on observe une différence d'environ $2 \%$ pour le français et pour l'anglais d'une année scolaire à l'autre. Il s'agit d'une hausse pour 
Moldavie et langue française, une relation séculaire et privilégiée

O. TURCAN

POLITIQUE, DÉFENSE ET REPRÉSENTATIONS l'anglais et d'une baisse pour le français.

En même temps, on observe une rupture visible avec les positions antérieures, où les textes officiels des premières années après l'obtention de l'indépendance ne comportaient aucune mention à l'égard d'une langue étrangère précise, mais des mesures pour les langues étrangères en général. En effet, plusieurs documents du pouvoir exécutif comme le Plan d'actions du Gouvernement pour les années 2012-2015 ou le Plan d'activité du Ministère de l'éducation pour 2013 se réfèrent dans leurs objectifs explicitement et uniquement au renforcement de l'enseignement de l'anglais.

Cette situation où l'enseignement du français est fragilisé en Moldavie et où l'anglais le devance, dans un contexte de mondialisation économique, peut ne pas surprendre. Pourtant, une des spécificités de la Moldavie par rapport aux autres États voisins et/ou européens, c'est le maintien du français en première position dans le classement général jusqu'en 2011. Pour comprendre les raisons du maintien du français et de son lent recul quantitatif, nous avons cherché des éléments de réponse auprès du Ministère de l'Éducation moldave, des acteurs éducatifs locaux, ainsi que des acteurs sociaux eux-mêmes (élèves, parents ou autres) qui peuvent l'influencer.

Si l'on examine les documents émis par les autorités éducatives centrales après 1991, on constate qu'il n'y a pas d'objectifs à moyen ou long terme qui concerneraient de manière explicite la langue française. Les mesures ou actions du pouvoir central en lien avec le français sont rares et souvent initiées par des acteurs externes de promotion du français présents en Moldavie (AUF, Ambassade de France, Alliance Française). Face à ce manque de volonté politique qui mène aux changements quantitatifs dans l'enseignement du français après 1991, certains acteurs du système éducatif adoptent une position de défense et de militantisme. Il se décline à travers différents discours selon l'époque:

a) entre 1991 et 2001, on observe un discours de défense du français par le biais du discours offensif contre l'anglais: «'invasion de l'anglais», «résister aux coups de force de l'anglais», «la bataille pour la francophonie», mener des actions «plus combatives»;

b) après 2001, on relève un discours de promotion du français pour la défense du plurilinguisme et de la diversité linguistique: le français contre le monolinguisme (anglais), contre l'uniformisation. Ce discours est influencé par les instruments du Conseil de l'Europe comme le Cadre européen commun de référence pour les langues, créé et diffusé en 
Moldavie et langue française, une relation séculaire et privilégiée

O. TURCAN
DU CÔTÉ DES ACTEURS SOCIAUX
2001. Quelques années plus tard, un autre document qui appuie ce discours du plurilinguisme en Moldavie est la Convention sur la protection et la promotion de la diversité des expressions culturelles de l'Unesco de 2005 (action appuyée par l'OIF), qui rappelle que «la diversité linguistique est un élément fondamental de la diversité culturelle». Ce discours devient de plus en plus fréquent autour de 2011, où le français et l'anglais arrivent à une situation d'équilibre quantitatif dans le cycle primaire et secondaire.

c) après 2011, où l'anglais dépasse le français par le nombre d'élèves qui l'étudie, les acteurs éducatifs évoquent le français comme un atout à côté de l'anglais.

À côté de la tradition et du militantisme en faveur du français, le nombre plus élevé des professeurs de français que celui des professeurs d'autres langues étrangères apparait comme une des raisons principales du maintien du français dans cet intervalle de vingt ans après l'indépendance.

L'examen des formations proposées aux futurs professeurs de français montre que les principales universités moldaves se fixent comme objectif d'observer les exigences du «marché du travail» et de s'adapter à la demande effectuée par les candidats à l'admission. Par conséquent, le français apparait de moins en moins dans les offres de formation et, à partir de 2005, il ne figure presque plus en tant que spécialité simple, mais dans des spécialités appelées «doubles» comme «français-anglais», «roumainfrançais», etc. La tendance du français à apparaître en deuxième position, tant dans les formations universitaires que dans les statistiques pour l'enseignement secondaire, s'accentue.

Il faut noter que ce n'est pas uniquement la quantité de professeurs de français, mais aussi la qualité de leur enseignement qui a contribué à la diffusion du français en Moldavie surtout après 1991. Ils ont joué un rôle important dans la transmission des connaissances ainsi que d'un «attachement au français», mentionné par les Moldaves participant à notre enquête sur le français en 2009. Et cela, pour une grande partie des professeurs, sans jamais avoir visité la France ou un autre pays francophone ou sans jamais rencontrer de Français ou parler avec un francophone [natif].

À côté des décideurs centraux et locaux de politique linguistique éducative et des professeurs de français, les acteurs sociaux (parents, élèves, étudiants ou autres) peuvent influencer la situation du français en Moldavie, à travers leurs choix et représentations du français. 
Moldavie et langue française, une relation séculaire et privilégiée

O. TURCAN
«QUE PENSEZ-VOUS DU FRANÇAIS?»
La question du choix du français nous a semblé importante pour l'explication de la place occupée par le français en Moldavie, à différents époques. Cette question sur la possibilité de choisir la langue étrangère à l'école a été posée dans le cadre de notre enquête sur la langue française, réalisée sur la base de deux questionnaires destinés à deux types de public: le «tout venant», c'està-dire tout Moldave locuteur ou non de français, et le public de l'Alliance française et de son réseau, bénéficiaire des activités que l'association organise. Au final, nous avons obtenu 87 témoignages, représentatifs d'un point de vue qualitatif. Une idée générale se dégage des réponses données par les informateurs: une des raisons de la présence et du maintien du français dans les écoles moldaves avant et peu après 1991 serait l'absence de la possibilité de choix d'une autre langue étrangère que celle qui était imposée dans l'enseignement, sauf dans la capitale ou éventuellement dans d'autres villes moldaves. Le français était en grande partie la seule langue étrangère enseignée dans les écoles. Selon un des témoignages, à cette époque-là, il y avait une sorte d'évidence, on ne se posait même pas la question d'un éventuel choix de langue: tout le monde apprenait le français.

Les témoignages des informateurs montrent également que dans les conditions où le choix existe et peut être exercé, le français va de pair avec l'anglais, la différence sur le marché de travail sera faite, selon eux, par le biais d'une troisième langue étrangère (l'italien, l'allemand, l'espagnol, etc.). Comme le note une informatrice, le plurilinguisme serait indispensable pour la «survie» économique des habitants des petits pays comme la Moldavie.

Les réponses à la question du choix et tout le discours que les enquêtés ont construit autour du choix des langues révèlent leurs représentations linguistiques. La question directe Que pensez-vous $d u$ français? a produit un discours sur la langue française, un discours constitutif de la situation du français, de son statut, de ses fonctions en Moldavie. Dans leurs réponses, la majorité de témoins ont adopté une approche esthétique: une langue (très) belle, mélodieuse, harmonieuse, douce (à l'oreille), élégante. Les rares témoins de notre enquête qui n'ont pas appris le français partagent le même avis et, parmi eux, des locuteurs du russe en tant que langue dite maternelle: F'aime beaucoup le français, c'est une langue mélodieuse et je la sens proche.

Le critère de la proximité linguistique ou de la parenté avec le roumain a été souvent mentionné: Le français est proche du roumain [...]. Dans la langue française, il y a beaucoup de mots qui ressemblent à 
Moldavie et langue française, une relation séculaire et privilégiée

O. TURCAN
RÉFÉRENCES BIBLIOGRAPHIQUES ceux du roumain...

Le français est considéré porteur d'une culture riche, prestigieuse - celle de la France et des Français. Plusieurs stéréotypes sont présents dans le discours de nombreux témoins. En effet, le français est associé à la ville de Paris et à ses monuments, à l'amour «langue des amoureux», «langue romantique».

Dans leur discours épilinguistique («discours sur»), certains témoins vont au-delà d'une sympathie pour le français. Ils révèlent leur fort attachement au français, une forme de lien affectif, quasiment irrationnel: J'aime (beaucoup), j'adore le français. Comment ne pas tomber amoureux du français? C'est une langue tellement belle!

Des différences générationnelles ressortent dans les réponses sur l'utilité et l'usage du français. Les informateurs de 13 à 19 ans ont, en grande partie, une perception plus pragmatique de l'apprentissage de la langue française (ou d'une langue étrangère, en général), orientée professionnellement. Ce public jeune semble avoir un contact plus étroit avec le milieu francophone naturel, en dehors des cours: usage du français lors des voyages, communication avec des Français. Cependant, dans les réponses des témoins âgés de 25 à 65 ans au moment de l'enquête, l'usage du français relève plutôt du culturel et de l'affectif: lecture en version originale des livres des écrivains français préférés, enrichir sa culture par le biais du français, apprendre le français pour le plaisir de le parler, etc.

Pour les jeunes générations, le français n'est plus perçu comme un but en soi ou une matière scolaire, comme il l'a été principalement pour les informateurs des générations d'avant 1991. Il est devenu un outil de communication dans des situations réelles, en dehors d'un cadre formel comme le cadre académique et un moyen pour accéder à des études universitaires relevant d'autres domaines que les spécialités linguistiques. Selon certains témoins, le français est une langue qui pourrait être nécessaire sur le marché du travail.

Cela étant dit, comment évoluera la place du français en Moldavie en l'absence d'une volonté politique en sa faveur? La tradition d'enseignement et l'attachement au français seront-ils suffisants pour son maintien? La Moldavie arrivera-t-elle à se placer avec le français à contre-courant d'une «modernité» globalisante?

BLANCHET P. 2012. La linguistique de terrain, méthode et théorie. Une approche ethnosociolinguistique de la complexité $\left(2^{\mathrm{e}}\right.$ éd.). Rennes, Presses universitaires de Rennes.

GUTU I. 2006. Aspects historiques de la francophonie moldave, 
Moldavie et langue française, une relation séculaire

et privilégiée

O. TURCAN pp. 36-40 in Actes du colloque international La Francopolyphonie comme vecteur de la communication, https://app.box.com/shared/5ifa4e0113

KLINKENBERG J.-M. 2000. Les politiques linguistiques: pour qui? pour quoi? pp. 99-114 in Demarty-Warzee J., Rousseau J. (dirs.). Les Cahiers du CIEP: Français de l'avenir et avenir du français. Paris: Didier.

PARMENTIER F. 2010. Moldavie. Les atouts de la francophonie. Paris: Éd. Non Lieu.

SALON A. 1981. L'Action culturelle de la France dans le monde: analyse critique. Thèse de doctorat, en 3 vol., Université Paris I PanthéonSorbonne.

TRUCHOT C. 2000. "L'espace externe" du français en Europe, pp. 71-81 in Demarty-Warzee J., Rousseau J. (dirs.). Les Cahiers du CIEP: Français de l'avenir et avenir du français. Paris: Didier.

TURCAN O. 2014. Le français en Moldavie: entre héritage, tradition et mondialisation. Thèse de doctorat sous la dir. de D. Huck (Université de Strasbourg) \& d'A. Gutu (Université Libre Internationale de Moldavie). 